PROCEEDINGS OF THE

AMERICAN MATHEMATICAL SOCIETY

Volume 133, Number 10, Pages 2939-2948

S 0002-9939(05)07883-4

Article electronically published on May 13, 2005

\title{
INTEGRABILITY OF SUBDIFFERENTIALS OF DIRECTIONALLY LIPSCHITZ FUNCTIONS
}

LIONEL THIBAULT AND NADIA ZLATEVA

(Communicated by Jonathan M. Borwein)

\begin{abstract}
Using a quantitative version of the subdifferential characterization of directionally Lipschitz functions, we study the integrability of subdifferentials of such functions over arbitrary Banach space.
\end{abstract}

\section{INTRODUCTION AND PRELIMINARIES}

The integration of subdifferentials concerns the problem of whether or not the condition that the subdifferential of $g$ contains the subdifferential of $f$ implies that $g$ and $f$ differ by a constant. The famous Rockafellar's integration result [9] states that the inclusion

$$
\partial f(x) \subset \partial g(x), \quad \text { for all } x \in X,
$$

implies that $g$ and $f$ are equal up to a constant whenever $f, g: X \rightarrow \mathbb{R} \cup\{+\infty\}$ are lower semicontinuous, proper, convex functions and $X$ is a Banach space. The result is established also for some classes of locally Lipschitz functions (see [1, 2, 4, 5]). An extension outside the convex and locally Lipschitz case is made by Poliquin [8] who showed that the integration result holds in a finite-dimensional setting for the functions $f$ and $g$ that are primal lower nice. Later, Thibault and Zagrodny [11] extended the result of Poliquin to the class of convexly subdifferentially similar functions defined on a Banach space. This class includes primal lower nice functions defined on a Hilbert space, as well as the differences of convex functions. We also refer to Ivanov and Zlateva [6] for the class of semi-convex functions. New insight on the subject can be found in [12, where a more general inclusion of subdifferentials is investigated.

None of the papers mentioned above deal with the case of directionally Lipschitz functions. The aim of the present paper is to study the integrability properties of directionally Lipschitz functions in order to find a natural extension of integrability results already established for Lipschitz functions. For the case of bivariate functions we refer to the paper of Thibault and Zlateva [13.

We begin by giving some necessary definitions and preliminaries. Further, we prove results concerning the subdifferential characterization of directionally Lipschitz property of a given function. We finish by establishing the local integrability

Received by the editors May 10, 2002.

2000 Mathematics Subject Classification. Primary 49J52; Secondary 28B20.

Key words and phrases. Subdifferential, integrability, directionally Lipschitz function.

The second author's research was supported by a Marie Curie fellowship of the European Community programme Improving Human Potential under contract No. HPMF-CT-2001-01345. 
of subdifferentials of strictly directionally Lipschitz regular functions, continuous on their domains (Theorem [3.3).

Throughout the paper $X$ is a real Banach space with open unit ball $B$ and topological dual $X^{*}$. For a function $g: X \rightarrow \mathbb{R} \cup\{+\infty\}$ we denote its effective domain by $\operatorname{dom} g:=\{x \in X: g(x)<+\infty\}$.

We will consider a general subdifferential operator $\partial$ that associates with each function $g: X \rightarrow \mathbb{R} \cup\{+\infty\}$ and with each point $x \in X$ a subset $\partial g(x)$ of $X^{*}$, that we will call a subdifferential of $g$ at $x$, and for which the following properties hold:

Property 1. $\partial g(x)=\emptyset$ if $x \notin \operatorname{dom} g$.

Property 2. $\partial g(x)=\partial f(x)$ whenever $g$ and $f$ coincide on a neighbourhood of $x$.

Property 3. $\partial g(x)$ is equal to the subdifferential in the sense of convex analysis whenever $g$ is convex.

Property 4. For $g$ lower semicontinuous near $x$ and $f$ convex and continuous on a neighbourhood of $x$, whenever $x \in \operatorname{dom}(f+g)$ is a local minimum point of $f+g$,

$$
0 \in \partial f(x)+\limsup _{y \rightarrow g} \partial g(y),
$$

where limsup denotes the weak star sequential limit superior and $y \rightarrow_{g} x$ means that $y \rightarrow x$ and $g(y) \rightarrow g(x)$.

Let us recall that all usual subdifferentials or presubdifferentials (see [11]) over appropriate Banach spaces are subdifferentials in the sense above. One of the most important subdifferentials is the well-known Clarke subdifferential. Supposing that $g$ is lower semicontinuous (in order to simplify), the Clarke subdifferential $\partial^{c} g(x)$ (see [10] and [3]) is equal to the set of all $x^{*} \in X^{*}$ such that for all $h \in X$

$$
\left\langle x^{*}, h\right\rangle \leq g^{\uparrow}(x ; h),
$$

where

$$
g^{\uparrow}(x ; h)=\sup _{\eta>0} \limsup _{\substack{y \rightarrow g \\ t \downarrow 0}} \inf _{\substack{t \in h+\eta B\\}} t^{-1}[g(y+t v)-g(y)] .
$$

Recall that the lower semicontinuous function $g: X \rightarrow \mathbb{R} \cup\{+\infty\}$ is said to be directionally Lipschitz at $x_{0} \in \operatorname{dom} g$ with respect to a vector $h_{0} \in X$ (see [10]), if there exist constants $K \in \mathbb{R}, \varepsilon>0, \delta>0$ such that

$$
\begin{aligned}
& t^{-1}[g(x+t h)-g(x)] \leq K, \\
& \forall t \in] 0, \varepsilon], \forall h \in h_{0}+\delta B, \forall x \in x_{0}+\delta B \text { with }\left|g(x)-g\left(x_{0}\right)\right| \leq \varepsilon .
\end{aligned}
$$

It is clear that $g$ is Lipschitz around $x_{0}$ exactly when it is directionally Lipschitz at $x_{0}$ with respect to $h_{0}=0$. Also, it is easy to see that in the case when the lower semicontinuous function $g$ considered in the definition above is convex, or continuous relative to its domain (i.e. for any $x_{0} \in \operatorname{dom} g$ and any $\gamma>0$ there exists $\eta>0$ such that $\left|g(x)-g\left(x_{0}\right)\right| \leq \gamma$ for all $x \in \operatorname{dom} g \cap\left(x_{0}+\eta B\right)$ ), then (1.1) is equivalent to the existence of constants $K \in \mathbb{R}, \varepsilon>0, \delta>0$ such that

$$
\begin{aligned}
& t^{-1}[g(x+t h)-g(x)] \leq K, \\
& \forall t \in] 0, \varepsilon], \forall h \in h_{0}+\delta B, \text { and } \forall x \in\left(x_{0}+\delta B\right) \cap \operatorname{dom} g .
\end{aligned}
$$

The lower semicontinuous function $g: X \rightarrow \mathbb{R} \cup\{+\infty\}$ is said to be (see [7]) strictly directionally Lipschitz at $x_{0} \in \operatorname{dom} g$ with respect to $h_{0} \in X$ if it is directionally Lipschitz at $x_{0}$ with respect to $h_{0}$ with some constants $K, \varepsilon, \delta$ satisfying (1.1) 
and, moreover,

$$
\begin{aligned}
& g \text { is locally Lipschitz on any set } x+] 0, \varepsilon]\left(h_{0}+\delta B\right), \\
& \text { where } x \in x_{0}+\delta B \text { and }\left|g(x)-g\left(x_{0}\right)\right| \leq \varepsilon
\end{aligned}
$$

If the lower semicontinuous function $g$ in the latter definition is also supposed to be continuous relative to its domain, or convex, then (1.3) can be replaced by

$$
\begin{aligned}
& g \text { is locally Lipschitz on any set } x+] 0, \varepsilon]\left(h_{0}+\delta B\right), \\
& \text { where } x \in\left(x_{0}+\delta B\right) \cap \operatorname{dom} g .
\end{aligned}
$$

Let us also recall that the lower semicontinuous function $g: X \rightarrow \mathbb{R} \cup\{+\infty\}$ is said to be regular at $x_{0} \in \operatorname{dom} g$ if $\liminf _{\substack{h^{\prime} \rightarrow h \\ t \downarrow 0}} t^{-1}\left[g\left(x_{0}+t h^{\prime}\right)-g\left(x_{0}\right)\right]=g^{\uparrow}\left(x_{0} ; h\right)$ for any $h \in X$, and it is said to be regular if it is regular at any point of its domain.

We wish to recall the Mean Value Theorem, established by Zagrodny in [15] (see also [1]), as it will be essentially used in what follows:

Theorem 1.1. Let $f: X \rightarrow \mathbb{R} \cup\{+\infty\}$ be a lower semicontinuous function, let $a, b \in \operatorname{dom} f$ with $a \neq b$ and let $\partial$ be any subdifferential operator. Then there exist $x_{n} \rightarrow_{f} c \in\left[a, b\left[:=\left\{(1-t) a+t b: t \in\left[0,1[\}\right.\right.\right.\right.$, and $x_{n}^{*} \in \partial f\left(x_{n}\right)$ such that:

(i) $f(b)-f(a) \leq \lim _{n \rightarrow \infty}\left\langle x_{n}^{*}, b-a\right\rangle$;

(ii) $\frac{\|b-c\|}{\|b-a\|}(f(b)-f(a)) \leq \lim _{n \rightarrow \infty}\left\langle x_{n}^{*}, b-x_{n}\right\rangle$;

(iii) $\|b-a\|(f(c)-f(a)) \leq\|c-a\|(f(b)-f(a))$.

\section{Subdifferential Properties of Directionally Lipschitz Functions}

In this section we study how the directionally Lipschitz property of the function refers to the properties of its subdifferential. Work in this direction with the Clarke subdifferential is that of Treiman [14], which is strongly based on the technique of Bishop and Phelps and on the result (see [14]) stating that $\liminf _{S \ni y \rightarrow x} K(S ; y) \subset T(S ; x)$ (here $K(S ;$.$) and T(S ;$.$) denote respectively the Bouligand contingent cone and$ the Clarke tangent cone of a closed subset $S \subset X)$. Our results are given in terms of arbitrary subdifferential, and their proofs are merely based on the Mean Value Theorem. They may also be considered as a quantitative version of [14], Theorem 6 . This quantitative version will be needed further to establish Lemma 3.1 that is a key step in our development.

In all the sequel $\partial$ stands for any subdifferential operator such that the corresponding subdifferential is included in the Clarke subdifferential, i.e., $\partial g(x) \subset$ $\partial^{c} g(x)$ for any function $g: X \rightarrow \mathbb{R} \cup\{+\infty\}$ and any $x \in X$.

Lemma 2.1. Assume that the lower semicontinuous function $g: X \rightarrow \mathbb{R} \cup\{+\infty\}$ is directionally Lipschitz at $x_{0}$ with respect to $h_{0}$ with constants $K, \varepsilon, \delta$ satisfying (1.1) (resp. (1.2)). Then

$$
\left\langle x^{*}, h_{0}\right\rangle+\delta\left\|x^{*}\right\| \leq K, \forall x^{*} \in \partial g(x), \forall x \in x_{0}+\delta B \text { with }\left|g(x)-g\left(x_{0}\right)\right| \leq \varepsilon,
$$

$$
\left.\left\langle x^{*}, h_{0}\right\rangle+\delta\left\|x^{*}\right\| \leq K, \forall x^{*} \in \partial g(x), \forall x \in x_{0}+\delta B\right) .
$$


Proof. Let $g: X \rightarrow \mathbb{R} \cup\{+\infty\}$ be directionally Lipschitz at $x_{0}$ with respect to $h_{0}$ with constants $K, \varepsilon, \delta$ satisfying (1.2) (resp. (1.1)). Then the condition (1.2) (resp. (1.1)) obviously implies

$$
\left.g^{\uparrow}(x ; h) \leq K, \forall h \in h_{0}+\delta B, \forall x \in x_{0}+\delta B \text { (with }\left|g(x)-g\left(x_{0}\right)\right| \leq \varepsilon\right) .
$$

Hence, if $\partial g(x) \neq \emptyset$ for some $x \in x_{0}+\delta B$ (with $\left|g(x)-g\left(x_{0}\right)\right| \leq \varepsilon$ ), then as $\partial g(x) \subset \partial^{c} g(x)$ we have

$$
\begin{gathered}
\left\langle x^{*}, h\right\rangle \leq g^{\uparrow}(x ; h) \leq K, \forall h \in h_{0}+\delta B, \forall x^{*} \in \partial g(x)\left(\text { with }\left|g(x)-g\left(x_{0}\right)\right| \leq \varepsilon\right), \text { i.e., } \\
\left\langle x^{*}, h_{0}\right\rangle+\delta\left\|x^{*}\right\| \leq K, \forall x^{*} \in \partial g(x), x \in x_{0}+\delta B\left(\text { with }\left|g(x)-g\left(x_{0}\right)\right| \leq \varepsilon\right) .
\end{gathered}
$$

We proceed to show the reverse implication, i.e., that (2.6) yields the property (1.2). As can be seen below, that case is more simple than the one establishing that (2.5) ensures the property (1.1). So, we made the choice of separating the two proofs.

Lemma 2.2. Let $g: X \rightarrow \mathbb{R} \cup\{+\infty\}$ be a lower semicontinuous function, let $x_{0} \in \operatorname{dom} g$ and let $h_{0} \in X$. Assume that there exist constants $K \in \mathbb{R}$ and $\delta>0$ such that (2.6) holds.

Then for any positive numbers $\varepsilon_{0}$ and $\delta_{0}$ such that $\varepsilon_{0}\left(\left\|h_{0}\right\|+\delta_{0}\right)+\delta_{0}<\delta$, property (1.2) holds for the function $g$ with $K, \varepsilon_{0}, \delta_{0}$.

Proof. Fix $\varepsilon_{0}>0$ and $\delta_{0}>0$ such that $\varepsilon_{0}\left(\left\|h_{0}\right\|+\delta_{0}\right)+\delta_{0}<\delta$ and take any $x \in\left(x_{0}+\delta_{0} B\right) \cap \operatorname{dom} g$, any $h \in h_{0}+\delta_{0} B$ with $h \neq 0$, and any $\left.\left.t \in\right] 0, \varepsilon_{0}\right]$. Consider $g(x+t h)$ and fix arbitrary real number $r \in \mathbb{R}$ with $r<g(x+t h)$. Define the lower semicontinuous function

$$
g_{r}(y):= \begin{cases}g(y), & \text { if } y \neq x+t h \\ r, & \text { if } y=x+t h .\end{cases}
$$

Apply the Mean Value Theorem of Zagrodny to estimate

$$
g_{r}(x+t h)-g_{r}(x)=r-g(x) \leq \lim _{n \rightarrow \infty}\left\langle x_{n}^{*}, t h\right\rangle
$$

with $x_{n}^{*} \in \partial g\left(x_{n}\right), x_{n} \underset{n \rightarrow \infty}{\longrightarrow} z \in\left[x, x+t h\left[, g\left(x_{n}\right) \underset{n \rightarrow \infty}{\longrightarrow} g(z)\right.\right.$. Then for $n$ large enough,

$$
\begin{aligned}
\left\|x_{n}-x_{0}\right\| & \leq\left\|x_{n}-z\right\|+\|z-x\|+\left\|x-x_{0}\right\| \leq\left\|x_{n}-z\right\|+t\|h\|+\delta_{0} \\
& \leq\left\|x_{n}-z\right\|+\varepsilon_{0}\left(\left\|h_{0}\right\|+\delta_{0}\right)+\delta_{0}<\delta
\end{aligned}
$$

Hence, $x_{n} \in x_{0}+\delta B$ and we can use (2.6) to get

$$
\begin{aligned}
\left\langle x_{n}^{*}, t h\right\rangle & =\left\langle x_{n}^{*}, t h_{0}\right\rangle+t\left\langle x_{n}^{*}, h-h_{0}\right\rangle \leq t\left[\left\langle x_{n}^{*}, h_{0}\right\rangle+\left\langle x_{n}^{*}, h-h_{0}\right\rangle\right] \\
& \leq t\left[\left\langle x_{n}^{*}, h_{0}\right\rangle+\delta_{0}\left\|x_{n}^{*}\right\|\right] \leq t K .
\end{aligned}
$$

This implies because of (2.7),

$$
r-g(x) \leq t K,
$$

which yields, on one hand, that $g(x+t h)$ is finite (i.e. $x+t h \in \operatorname{dom} g$ ) and, on the other hand, that

$$
g(x+t h)-g(x) \leq t K .
$$


Observe that the inequality also holds for $h=0$, in the case when $0 \in h_{0}+\delta_{0} B$ since $K \geq 0$ in that case because of (2.6) . Therefore, the property (1.2) holds for $g$ at $x_{0}$ with respect to $h_{0}$ with $K, \varepsilon_{0}, \delta_{0}$ as above.

Theorem 2.3 (subdifferential characterization of directionally Lipschitz property). Let $g: X \rightarrow \mathbb{R} \cup\{+\infty\}$ be a lower semicontinuous function, let $x_{0} \in \operatorname{dom} g$ and let $h_{0} \in X$. Then $g$ is directionally Lipschitz at $x_{0}$ with respect to $h_{0}$ with constants $K, \varepsilon^{\prime}, \delta^{\prime}$ satisfying (1.1), if and only if there exist constants $\varepsilon>0$ and $\delta>0$ such that (2.5) holds with $K, \varepsilon, \delta$.

Proof. The implication $\Longrightarrow$ follows from Lemma 2.1.

Let us prove the converse.

Without loss of generality we may suppose that $\varepsilon \in] 0,1[$ and $\delta \in] 0, \varepsilon[$, and that they are such that

$$
g\left(x_{0}\right) \leq g(x)+\varepsilon, \forall x \in x_{0}+\delta B .
$$

Now take $\varepsilon^{\prime}>0$ such that $\varepsilon^{\prime}\left(\left\|h_{0}\right\|+|K|+2\right)<\delta$, and $\left.\delta^{\prime} \in\right] 0, \varepsilon^{\prime}\left[\right.$. Fix any $x \in x_{0}+\delta^{\prime} B$ with $\left|g(x)-g\left(x_{0}\right)\right| \leq \varepsilon^{\prime}$, any $h \in h_{0}+\delta^{\prime} B$ with $h \neq 0$, and any $\left.\left.t \in\right] 0, \varepsilon^{\prime}\right]$. Consider $g(x+t h)$ and suppose $g(x+t h)>g(x)+t K$. We may choose and fix a real number $\mu \in] 0,1[$ such that $g(x+t h)>g(x)+t(K+\mu)$. Set $r:=g(x)+t(K+\mu)$ and define the lower semicontinuous function

$$
g_{r}(y):= \begin{cases}g(y), & \text { if } y \neq x+t h \\ r, & \text { if } y=x+t h .\end{cases}
$$

Apply the Mean Value Theorem of Zagrodny to estimate

$$
g_{r}(x+t h)-g_{r}(x)=r-g(x) \leq \lim _{n \rightarrow \infty}\left\langle x_{n}^{*}, t h\right\rangle
$$

with some sequences $x_{n}^{*} \in \partial g\left(x_{n}\right), x_{n} \underset{n \rightarrow \infty}{\longrightarrow} z \in\left[x, x+t h\left[, g\left(x_{n}\right) \underset{n \rightarrow \infty}{\longrightarrow} g(z)\right.\right.$. Then, for $n$ large enough, $\left\|x_{n}-x_{0}\right\| \leq\left\|x_{n}-z\right\|+\varepsilon^{\prime}\left(\left\|h_{0}\right\|+\delta^{\prime}\right)+\delta^{\prime}<\delta$.

CASE I. $z=x$.

Then $g\left(x_{n}\right) \underset{n \rightarrow \infty}{\longrightarrow} g(x)$ and for $n$ large enough

$$
\left|g\left(x_{n}\right)-g\left(x_{0}\right)\right| \leq\left|g\left(x_{n}\right)-g(x)\right|+\left|g(x)-g\left(x_{0}\right)\right| \leq\left|g\left(x_{n}\right)-g(x)\right|+\varepsilon^{\prime}<\varepsilon .
$$

CASE II. $z \neq x$.

Then Mean Value Theorem of Zagrodny and in particular (iii) ensures that

$$
t\|h\|(g(z)-g(x)) \leq\|z-x\|(r-g(x)) .
$$

We rewrite the left-hand side

$$
\begin{aligned}
t\|h\|\left(g(z)-g\left(x_{0}\right)\right) & \leq\|z-x\|(r-g(x))+t\|h\|\left(g(x)-g\left(x_{0}\right)\right) \\
& \leq\|z-x\|(r-g(x))+t\|h\| \varepsilon^{\prime} \\
& \leq t\|h\|\left(r-g(x)+\varepsilon^{\prime}\right),
\end{aligned}
$$

from where

$$
\begin{aligned}
g(z)-g\left(x_{0}\right) & \leq r-g(x)+\varepsilon^{\prime}=t(K+\mu)+\varepsilon^{\prime} \\
& \leq t(|K|+\mu)+\varepsilon^{\prime} \leq \varepsilon^{\prime}(|K|+\mu+1) \leq \varepsilon^{\prime}(|K|+2) .
\end{aligned}
$$

Hence, for sufficiently large $n$ we have

$$
g\left(x_{n}\right)-g\left(x_{0}\right)=g\left(x_{n}\right)-g(z)+g(z)-g\left(x_{0}\right) \leq\left|g\left(x_{n}\right)-g(z)\right|+\varepsilon^{\prime}(|K|+2)<\varepsilon .
$$


Obviously, for $n$ large enough, the points $x_{n} \in x_{0}+\delta B$ and hence by (2.8) we also have that $g\left(x_{0}\right)-g\left(x_{n}\right) \leq \varepsilon$. Therefore, we obtain $\left|g\left(x_{n}\right)-g\left(x_{0}\right)\right| \leq \varepsilon$.

In both cases one has for $n$ large enough, $x_{n} \in x_{0}+\delta B$ and $\left|g\left(x_{n}\right)-g\left(x_{0}\right)\right| \leq \varepsilon$, then by (2.5) one has $\left\langle x_{n}^{*}, h\right\rangle \leq K$. So, by 2.9

$$
r-g(x) \leq t K \text {, i.e., } t(K+\mu) \leq t K,
$$

and the latter yields $\mu \leq 0$, which is a contradiction, since $\mu>0$. We conclude that $g(x+t h) \leq g(x)+t K$, and further, if $0 \in h_{0}+\delta^{\prime} B$, the inequality still holds for $h=0$ because, according to (2.5), $K \geq 0$ in that case. The proof is then complete.

\section{LOCAL INTEGRABILITY}

In this section we use the results proved in the previous one to establish the local integrability of any subdifferential of a class of directionally Lipschitz functions.

We begin by showing how property (1.2) is implied by inclusion of subdifferentials for lower semicontinuous functions.

Lemma 3.1. Let $g: X \rightarrow \mathbb{R} \cup\{+\infty\}$ be a lower semicontinuous function which satisfies property (1.2) at $x_{0} \in \operatorname{dom} g$ with respect to $h_{0} \in X$ with some constants $K, \varepsilon, \delta$. Let $f: X \rightarrow \mathbb{R} \cup\{+\infty\}$ be a lower semicontinuous function such that $\partial f(x) \subset \partial g(x)$ for any $x \in x_{0}+\delta B$.

Then for any constants $\delta_{0}>0$ and $\varepsilon_{0}>0$ such that $\delta_{0}+\varepsilon_{0}\left(\left\|h_{0}\right\|+\delta_{0}\right)<\delta$ with $\operatorname{dom} f \cap\left(x_{0}+\delta_{0} B\right) \neq \emptyset$, one has that property (1.2) holds for $f$ with the constants $K, \varepsilon_{0}, \delta_{0}$, i.e.,

$\left.\left.t^{-1}[f(x+t h)-f(x)] \leq K, \forall t \in\right] 0, \varepsilon_{0}\right], \forall h \in h_{0}+\delta_{0} B$, and $\forall x \in\left(x_{0}+\delta_{0} B\right) \cap \operatorname{dom} f$. In particular, $x+\left[0, \varepsilon_{0}\right]\left(h_{0}+\delta_{0} B\right) \subset \operatorname{dom} f$, for all $x \in \operatorname{dom} f \cap\left(x_{0}+\delta_{0} B\right)$. Further, $f$ is directionally Lipschitz at $x_{0}$ with respect to $h_{0}$, whenever $x_{0} \in \operatorname{dom} f$.

Proof. Suppose that $g$ satisfies (1.2) with constants $K, \varepsilon, \delta$. By Lemma 2.1 for any $x \in \operatorname{dom} g \cap\left(x_{0}+\delta B\right)$ and any $x^{*} \in \partial g(x)$ we have $\left\langle x^{*}, h_{0}\right\rangle+\delta\left\|x^{*}\right\| \leq K$. Because of the assumption $\partial f(x) \subset \partial g(x)$ for any $x \in x_{0}+\delta B$, that inequality holds in particular for all $x \in \operatorname{dom} \partial f \cap\left(x_{0}+\delta B\right)$ and $x^{*} \in \partial f(x)$.

Fix any $\delta_{0}>0$ and $\varepsilon_{0}>0$ such that $\delta_{0}+\varepsilon_{0}\left(\left\|h_{0}\right\|+\delta_{0}\right)<\delta$. Lemma 2.2 ensures that for all $\left.\left.x \in \operatorname{dom} f \cap\left(x_{0}+\delta_{0} B\right), t \in\right] 0, \varepsilon_{0}\right]$, and $h \in h_{0}+\delta_{0} B$ one has $t^{-1}[f(x+t h)-f(x)] \leq K$. The proof is then complete.

We will also need the following second lemma of general interest. It concerns the graphical density of the domain of any subdifferential in the sense of the first section.

Lemma 3.2. Let $f: X \rightarrow \mathbb{R} \cup\{+\infty\}$ be a lower semicontinuous function with $\operatorname{dom} f \neq \emptyset$. Then $\operatorname{dom} \partial f$ is $f$-graphically dense in $\operatorname{dom} f$, i.e., for any $a \in \operatorname{dom} f$ there exists a sequence $x_{n} \in \operatorname{dom} \partial f$ with $x_{n} \underset{n \rightarrow \infty}{\longrightarrow}$ and $f\left(x_{n}\right) \underset{n \rightarrow \infty}{\longrightarrow} f(a)$.

Proof. Fix $a \in \operatorname{dom} f$ and $\varepsilon>0$. Choose by the lower semicontinuity of $f$ some positive number $r<\varepsilon$ such that $f(x)>f(a)-\varepsilon$ for all $x \in a+r B$. If for any $b \in a+r B$ one has $f(b) \geq f(a)$, then $a$ is a local minimum point of $f$ and by Property 4 we have that

$$
0 \in \limsup _{x_{n} \rightarrow f_{f} a} \partial f\left(x_{n}\right),
$$


i.e., there exist $x_{n} \in \operatorname{dom} \partial f$ such that $x_{n} \underset{n \rightarrow \infty}{\longrightarrow} a$ and $f\left(x_{n}\right) \underset{n \rightarrow \infty}{\longrightarrow} f(a)$. Otherwise, there exists some $b \in a+r B$ with $f(b)<f(a)$ and the Mean Value Theorem of Zagrodny yields $x_{n} \underset{n \rightarrow \infty}{\longrightarrow} c \in\left[a, b\left[\right.\right.$ with $f\left(x_{n}\right) \underset{n \rightarrow \infty}{\longrightarrow} f(c)$ and $\partial f\left(x_{n}\right) \neq \emptyset$, and such that conclusion (iii) of that theorem holds. The latter gives $\lim _{n \rightarrow \infty} f\left(x_{n}\right)=f(c) \leq f(a)$. We deduce the existence of some $N$ such that $\left\|x_{n}-a\right\|<r<\varepsilon$ and $\left|f\left(x_{n}\right)-f(a)\right|<\varepsilon$ for all $n \geq N$, and hence the proof is complete.

We establish now the integrability result.

Theorem 3.3 (integrability of regular directionally Lipschitz functions). Let $g$ : $X \rightarrow \mathbb{R} \cup\{+\infty\}$ be a lower semicontinuous regular function, continuous relative to its domain, and strictly directionally Lipschitz at $x_{0} \in \operatorname{dom} g$.

Then there exist constants $\alpha>0$ and $\beta \in] 0, \alpha[$ such that for any lower semicontinuous function $f: X \rightarrow \mathbb{R} \cup\{+\infty\}$ with $\operatorname{dom} f \cap\left(x_{0}+\beta B\right) \neq \emptyset$, the inclusion $\partial f(x) \subset \partial g(x)$ for all $x \in x_{0}+\alpha B$ implies that

$$
f=g+\text { const } \quad \text { on } x_{0}+\beta B .
$$

If the strict directionally Lipschitz property for $g$ at $x_{0}$ with respect to $h_{0}$ holds with constants $K, \varepsilon, \delta$ satisfying $(1.2)$, one may take $\alpha=\delta$ and $\beta=\min \left\{\frac{\delta^{2}}{4\left(\left\|h_{0}\right\|+2 \delta\right)}, \frac{\varepsilon \delta}{2}\right\}$.

Proof. Let $K, \varepsilon$, and $\delta$ be given by the strictly directionally Lipschitz property of $g$ at $x_{0}$ with respect to $h_{0}$, i.e., properties (1.2) and (1.4) hold for them.

Set $\delta_{0}:=\frac{\delta}{2}$ and $\varepsilon_{0}:=\min \left\{\frac{\delta}{2\left(\left\|h_{0}\right\|+2 \delta\right)}, \varepsilon\right\}$, and observe that $\varepsilon_{0}<1$. It is easy to see that the conclusion of Lemma 3.1 holds for such $\delta_{0}$ and $\varepsilon_{0}$.

Put now $\alpha:=\delta$ and $\beta:=\varepsilon_{0} \delta_{0}=\min \left\{\frac{\delta^{2}}{4\left(\left\|h_{0}\right\|+2 \delta\right)}, \frac{\varepsilon \delta}{2}\right\}$.

We claim that for arbitrary $x \in x_{0}+\beta B$ there exists $h_{x} \in h_{0}+\frac{\delta}{2} B$ such that

$$
x+\varepsilon_{0} h_{x}=x_{0}+\varepsilon_{0} h_{0} .
$$

Indeed, for $x \in x_{0}+\beta B$ we may set $h_{x}:=\frac{x_{0}-x}{\varepsilon_{0}}+h_{0}$ and since $\frac{\left\|x-x_{0}\right\|}{\varepsilon_{0}}<\frac{\beta}{\varepsilon_{0}}=$ $\frac{\varepsilon_{0} \delta_{0}}{\varepsilon_{0}}=\frac{\delta}{2}$, we obtain that $h_{x} \in h_{0}+\frac{\delta}{2} B$.

Moreover, the inclusion $h_{x} \in h_{0}+\frac{\delta}{2} B$ ensures that

$$
\left.\left.\left.x+] 0, \varepsilon_{0}\right]\left(h_{x}+\frac{\delta}{2} B\right) \subset x+\right] 0, \varepsilon\right]\left(h_{0}+\delta B\right), \quad \forall x \in x_{0}+\beta B .
$$

Now, fix arbitrary $v \in \operatorname{dom} f \cap\left(x_{0}+\beta B\right)$ which is a non-empty set by assumption. As the set $\operatorname{dom} \partial f$ is $f$-graphically dense in $\operatorname{dom} f$ by Lemma [3.2, we obtain a sequence $\left\{x_{n}\right\} \subset \operatorname{dom} \partial f \subset \operatorname{dom} \partial g \subset \operatorname{dom} g$, such that $x_{n} \underset{n \rightarrow \infty}{\longrightarrow} v$ and $f\left(x_{n}\right) \underset{n \rightarrow \infty}{\longrightarrow} f(v)$. Writing

$$
\left\|x_{n}-x_{0}\right\| \leq\left\|x_{n}-v\right\|+\left\|v-x_{0}\right\|<\left\|x_{n}-v\right\|+\beta,
$$

we see that for sufficiently large $n$ (for example $n \geq N_{1}$ ) we have

$$
\left\|x_{n}-x_{0}\right\|<\beta .
$$

For $n \geq N_{1}$ denote by $C_{n}$ the open convex set $\left.\left.C_{n}:=x_{n}+\right] 0, \varepsilon_{0}\right]\left(h_{x_{n}}+\frac{\delta}{2} B\right)$. Recall that for $n \geq N_{1}$ we have $x_{n} \in\left(x_{0}+\beta B\right) \cap \operatorname{dom} g$ and observe also, by what precedes, that $h_{x_{n}} \in h_{0}+\frac{\delta}{2} B$. So, by the definition of strictly directionally Lipschitz property of $g$ and by (3.11) it follows that $g$ is locally Lipschitz and regular on $C_{n}$. From Lemma 3.1 it is clear that $\operatorname{dom} f \cap C_{n} \neq \emptyset$ for $n \geq N_{1}$, since $\left.\left.x_{n}+\right] 0, \varepsilon_{0}\right] h_{x_{n}} \subset \operatorname{dom} f$. 
It remains to observe that the inclusion of subdifferentials holds on $C_{n}$ for $n \geq$ $N_{1}$, since $C_{n} \subset x_{0}+\alpha B$. Indeed, for any $x \in C_{n}$ we may write $x:=x_{n}+t\left(h_{x_{n}}+p\right)$ for some $\left.t \in] 0, \varepsilon_{0}\right]$ and some $p \in \frac{\delta}{2} B$ which ensures by (3.12) that

$$
\begin{aligned}
\left\|x-x_{0}\right\| & =\left\|x_{n}+t\left(h_{x_{n}}+p\right)-x_{0}\right\| \leq\left\|x_{n}-x_{0}\right\|+t\left\|h_{x_{n}}+p\right\| \\
& <\beta+\varepsilon_{0}\left(\left\|h_{0}\right\|+\frac{\delta}{2}+\frac{\delta}{2}\right)=\varepsilon_{0} \frac{\delta}{2}+\varepsilon_{0}\left(\left\|h_{0}\right\|+\delta\right) \\
& =\varepsilon_{0}\left(\left\|h_{0}\right\|+\frac{3 \delta}{2}\right) \leq \frac{\delta}{2\left(\left\|h_{0}\right\|+2 \delta\right)}\left(\left\|h_{0}\right\|+\frac{3 \delta}{2}\right)<\delta=\alpha .
\end{aligned}
$$

Hence, we can apply the integrability result for locally Lipschitz regular functions (see [4] and Theorem 4.1 in [12]) to obtain that there exists some real constant $c_{n}$ such that

$$
f(x)=g(x)+c_{n}, \quad \forall x \in C_{n}, \quad \forall n \geq N_{1} .
$$

From (3.10) we have that $x_{n}+\varepsilon_{0} h_{x_{n}}=x_{0}+\varepsilon_{0} h_{0} \in C_{n}$, for any $n \geq N_{1}$, hence

$$
f\left(x_{0}+\varepsilon_{0} h_{0}\right)=g\left(x_{0}+\varepsilon_{0} h_{0}\right)+c_{n}, \quad \forall n \geq N_{1},
$$

and, in particular because $g\left(x_{0}+\varepsilon_{0} h_{0}\right)$ is finite according to (1.2), the value of $c_{n}$ does not depend on $n$ for $n \geq N_{1}$, say $c_{n}=c=f\left(x_{0}+\varepsilon_{0} h_{0}\right)-g\left(x_{0}+\varepsilon_{0} h_{0}\right)$, for all $n \geq N_{1}$, i.e.,

$$
f(x)=g(x)+c, \quad \forall x \in C_{n}, \quad \forall n \geq N_{1} .
$$

Now we proceed to prove the equality $f(v)=g(v)+c$.

First, let us show that $f\left(x_{n}\right)=g\left(x_{n}\right)+c$ for $n \geq N_{1}$. Observing by the definition of $C_{n}$ that for some vectors $h_{0}$ one may have $x_{n} \notin C_{n}$, we begin by verifying that $f\left(x_{n}+t h_{x_{n}}\right) \underset{t \downarrow 0}{\longrightarrow} f\left(x_{n}\right)$.

As $f$ is lower semicontinuous, we always have that $f\left(x_{n}\right) \leq \liminf _{t \downarrow 0} f\left(x_{n}+t h_{x_{n}}\right)$. Further, for $\left.t \in] 0, \varepsilon_{0}\right]$, Lemma 3.1 gives that $x_{n}+t h_{x_{n}} \in \operatorname{dom} f$ and $f\left(x_{n}+t h_{x_{n}}\right)-$ $f\left(x_{n}\right) \leq K t$. Hence, $\limsup f\left(x_{n}+t h_{x_{n}}\right) \leq f\left(x_{n}\right)$ and the claim is proved.

Analogously, using the strictly directionally Lipschitz property of $g$ at $x_{0}$ with respect to $h_{0}$ along with its lower semicontinuity, it is not difficult to see that $g\left(x_{n}+t h_{x_{n}}\right) \underset{t \downarrow 0}{\longrightarrow} g\left(x_{n}\right)$, and then, recalling that $f\left(x_{n}\right)$ and $g\left(x_{n}\right)$ are finite because of $x_{n} \in \operatorname{dom} \partial f \subset \operatorname{dom} \partial g$, we may conclude that

$$
(f-g)\left(x_{n}\right)=\lim _{t \downarrow 0}(f-g)\left(x_{n}+t h_{x_{n}}\right)=c, \quad \forall n \geq N_{1} .
$$

As $x_{n} \underset{n \rightarrow \infty}{\longrightarrow} v$, the lower semicontinuity of $g$ implies that

$$
g(v) \leq \liminf _{n \rightarrow \infty} g\left(x_{n}\right)=\lim _{n \rightarrow \infty} f\left(x_{n}\right)-c=f(v)-c,
$$

in particular, $v \in \operatorname{dom} g$, hence $v \in \operatorname{dom} g \cap\left(x_{0}+\beta B\right)$. The continuity of $g$ relative to its domain ensures that $g(v)=\lim _{n \rightarrow \infty} g\left(x_{n}\right)$. By (3.14) and the fact that $f\left(x_{n}\right) \underset{n \rightarrow \infty}{\longrightarrow} f(v)$ we obtain

$$
g(v)=\lim _{n \rightarrow \infty} g\left(x_{n}\right)=\lim _{n \rightarrow \infty}\left[f\left(x_{n}\right)-c\right]=f(v)-c,
$$

i.e. $f(v)=g(v)+c$. 
Hence we have proved that

$$
f(v)=g(v)+c, \quad \forall v \in \operatorname{dom} f \cap\left(x_{0}+\beta B\right),
$$

and at the same time we obtain via that equality

$$
\operatorname{dom} f \cap\left(x_{0}+\beta B\right) \subset \operatorname{dom} g \cap\left(x_{0}+\beta B\right) .
$$

To finish the proof, it remains only to establish the opposite inclusion of the domains. Take arbitrary $u \in \operatorname{dom} g \cap\left(x_{0}+\beta B\right)$ and set $\left.\left.C:=u+\right] 0, \varepsilon_{0}\right]\left(h_{u}+\frac{\delta}{2} B\right)$. Note that for any $x \in \operatorname{dom} f \cap\left(x_{0}+\beta B\right) \neq \emptyset$, the point $x+\varepsilon_{0} h_{x} \in \operatorname{dom} f$ by Lemma 3.1 and, moreover,

$$
x+\varepsilon_{0} h_{x}=x_{0}+\varepsilon_{0} h_{0}=u+\varepsilon_{0} h_{u} .
$$

This ensures that $\operatorname{dom} f \cap C \neq \emptyset$. The assumptions of [12], Theorem 4.1 hold for $f, g$ and $C$, and we apply it to conclude that

$$
f(x)=g(x)+c, \quad \forall x \in C .
$$

The constant is still $c=f\left(x_{0}+\varepsilon_{0} h_{0}\right)-g\left(x_{0}+\varepsilon_{0} h_{0}\right)$ because by (3.15) one has $x_{0}+\varepsilon_{0} h_{0} \in C$. Observe that for any $\left.\left.t \in\right] 0, \varepsilon_{0}\right]$ the points $u+t h_{u} \in C \subset \operatorname{dom} g$, where the last inclusion holds because of (1.2). Using the lower semicontinuity of $f$ at $u$, and the continuity of $g$ with respect to its domain, we obtain that

$$
f(u) \leq \liminf _{t \downarrow 0} f\left(u+t h_{u}\right)=\liminf _{t \downarrow 0} g\left(u+t h_{u}\right)+c=g(u)+c,
$$

hence, $u \in \operatorname{dom} f$. The proof is then complete.

Note that the continuity assumption of the restriction of $g$ on $V \cap \operatorname{dom} g$ (where $V$ is some neighbourhood of $x_{0}$ ) is crucial. It suffices to consider the function $g$ from $\mathbb{R}$ into $\mathbb{R}$ with $g(x)=0$ if $x \geq 0$ and $g(x)=1$ if $x<0$ and the function $f$ from $\mathbb{R}$ into $\mathbb{R}$ with $f(x)=0$ if $x \geq 0$ and $f(x)=2$ if $x<0$. We have $\partial f(x) \subset \partial g(x)$ for all $x \in \mathbb{R}$ but the functions $f$ and $g$ are not equal near 0 up to a constant.

In the same way, it is easily seen that the lower semicontinuity assumption of $f$ is also essential.

\section{REFERENCES}

[1] J. Borwein, W. Moors, Essentially smooth Lipschitz functions, J. Funct. Anal. 149 (1997), No. 2, 305-351. MR1472362 (98i:58028)

[2] J. Borwein, W. Moors, X. Wang, Generalized subdifferentials: a Baire categorical approach, Trans. Amer. Math. Soc. 353 (2001), No. 10, 3875-3893. MR1837212 (2002f:49026)

[3] F. Clarke, Optimization and nonsmooth analysis, New York, John Wiley \& Sons (1983). MR0709590 (85m:49002)

[4] R. Correa, A. Jofre, Tangentially continuous directional derivatives in nonsmooth analysis, J. Optim. Theory Appl. 61 (1989), 1-21. MR0993912 (90h:49009)

[5] R. Correa, L. Thibault, Subdifferential analysis of bivariate separate regular functions, J. Math. Anal. Appl. 148 (1990), 157-174. MR1052052 (91b:49018)

[6] M. Ivanov, N. Zlateva, Abstract subdifferential calculus and semi-convex functions, Serdica Math. J. 23 (1997), 35-58. MR.1610289 (99f:49020)

[7] A. Jofre, L. Thibault, D-representation of subdifferentials of directionally Lipschitz functions, Proc. Amer. Math. Soc. 110 (1990), No. 1, 117-123. MR1015680 (90m:90302)

[8] R. Poliquin, Subgradient monotonicity and convex functions, Nonlinear Anal. 17 (1990), 305-317. MR1040008 (91b:90155)

[9] R. T. Rockafellar, On the maximal monotonicity of subdifferential mappings, Pacific J. Math. 33 (1970), 209-216. MR0262827 (41:7432)

[10] Generalized directional derivatives and subgradients of nonconvex functions, Canad. J. Math. 32 (1980), No. 2, 257-280. MR0571922|(81f:49006) 
[11] L. Thibault, D. Zagrodny, Integration of subdifferentials of lower semicontinuous functions on Banach spaces, J. Math. Anal. Appl. 189 (1995), 33-58. MR.1312029 (95i:49032)

[12] _ Enlarged inclusion of subdifferentials, Canad. Math. Bull., to appear.

[13] L. Thibault, N. Zlateva, Integrability of subdifferentials of certain bivariate functions, Nonlinear Anal. 54 (2003), 1251-1269. MR1995929 (2004m:49052)

[14] J. Treiman, Generalized gradients, Lipschitz behaviour and directional derivatives, Canad. J. Math. 37 (1985), No. 6, 1074-1084. MR0828835 (87h:90263)

[15] D. Zagrodny, Approximate mean value theorem for upper subderivatives, Nonlinear Anal. 12 (1988), 1413-1428. MR0972409 (89k:58034)

Département de Mathématiques, Université Montpellier II, CC 051, Place Eugène Bataillon, 34095 Montpellier cedex 5, France

E-mail address: thibault@math.univ-montp2.fr

Section of Operations Research, Department of Mathematics, Sofia University, 5. James Bourchier Blvd., 1164 Sofia, Bulgaria - and - Département de Mathématiques, Université Montpellier II, CC 051, Place Eugène Bataillon, 34095 Montpellier CedeX 5, France

E-mail address: zlateva@fmi.uni-sofia.bg

E-mail address: zlateva@math.univ-montp2.fr 